\title{
recillunds
}

Revista Cientifica Mundo de la Investigación y el Conocimiento

Félix Arquímides Valdez Rivera ${ }^{a}$

La seguridad ciudadana frente al derecho a la defensa en libertad de los procesados

Citizen security against the right to defense in freedom of the defendants

Revista Científica Mundo de la Investigación y el Conocimiento. Vol. 3 núm. 4., diciembre, ISSN: 2588-073X, 2019, pp. 39-77

DOI: 10.26820/recimundo/3.(4).diciembre.2019.39-77

URL: http://recimundo.com/index.php/es/article/view/652

Código UNESCO: 5605 Legislación y Leyes Nacionales

Tipo de Investigación: Artículo de Revisión

(C) RECIMUNDO; Editorial Saberes del Conocimiento, 2019

Recibido: 15/09/2019

Aceptado: 23/11/2019

Publicado: 30/12/2019

Correspondencia: ab.felixval25@hotmail.com

a. Magister en Derecho Constitucional; Abogado de los Tribunales y Juzgados de la Republica del Ecuador; Licenciado en Ciencias Sociales y Políticas; Investigador Independiente; Guayaquil, Ecuador; ab.felixval25@hotmail.com 


\section{La seguridad ciudadana frente al derecho a la defensa en libertad de los procesados}

Vol. 3, núm. 4., (2019)

Félix Arquímides Valdez Rivera

\section{RESUMEN}

La investigación que se presenta tiene por intención, en primer lugar, llevar a cabo un análisis de la evolución, la implementación y el significado del monitoreo telemático, así como su instrumentación en la República del Ecuador, partiendo del reconocimiento de la problemática siguiente: ¿Cómo contribuir a la preservación de la paz social en el ámbito de la introducción de medidas sustitutivas como la vigilancia electrónica de personas privadas de libertad?, tomando en cuenta su aparición para complementar y reforzar dos medidas sustantivas a la privación de libertad: reclusión parcial y libertad vigilada intensiva. Así, la investigación persigue revelar la finalidad de la pulsera electrónica y su introducción en la sociedad ecuatoriana, a partir de las dinámicas de debate público, en cuanto a su implementación.

Asumiendo como metodología el Derecho Comparado y su trascendencia al valorar contextos jurídicos diversos. En este sentido, busca evidenciar los objetivos y funciones de tal medida y sus implicaciones a nivel estatal y social. Al propio tiempo cuestiona la aplicación del instrumento, constituyéndose como un mecanismo de vigilancia, normalización y control del cuerpo del individuo, que asume el fracaso de la rehabilitación en la prisión y centra sus efectos simbólicos en la violencia hacia el sujeto y en el control de la sociedad tan propio de la época postmodernista.

Se sostiene, que esta medida que representa el ideal privatizador de la justicia, propio del Workface, por medio de la instalación de un discurso carcelario y empresarial en las prácticas punitivas del Estado, esto en virtud de la implementación de procesos que son utilizados en los grandes emporios empresariales. Valorándose su impacto en la sociedad.

Palabras Claves: Monitoreo Telemático Judicial; Medidas Sustitutivas a la Privación de Libertad; Control; Vigilancia; Seguridad Ciudadana. 


\title{
La seguridad ciudadana frente al derecho a la defensa en libertad de los \\ procesados
}

Vol. 3, núm. 4., (2019)

Félix Arquímides Valdez Rivera

\begin{abstract}
The communication presented is intended, first, to conduct an analysis of the development, implementation and meaning of telematic monitoring and implementation in the Republic of Ecuador, based on recognition of the following problems: How to contribute to the preservation of social peace in the area of the introduction of alternative measures such as electronic surveillance of persons deprived of liberty ?, taking into account its appearance to complement and reinforce two substantive deprivation of liberty: partial seclusion and intensive probation. Thus, the research aims to reveal the purpose of the electronic bracelet and its introduction into Ecuadorian society, from the dynamics of public debate regarding its implementation.

Assuming as comparative law methodology and its importance in assessing various legal contexts. In this sense, it seeks to show the objectives and functions of such a measure and its implications at the state and social level. At the same time challenging the application of the instrument, valuing constitutes a mechanism for monitoring, standardization and control of the individual's body, which assumes the failure to rehabilitate prison and focuses its symbolic effects on violence towards the subject and control society postmoder so characteristic of the era.

It is argued that this measure representing the privatization ideal of justice, the workface own, through the installation of a business discourse in prison and punitive practices of the State. Assessing how society.
\end{abstract}

Keywords: Telematic Monitoring Judicial Alternative to Deprivation of Liberty; Control; Surveillance; Public Safety. 


\section{La seguridad ciudadana frente al derecho a la defensa en libertad de los procesados}

Vol. 3, núm. 4., (2019)

Félix Arquímides Valdez Rivera

\section{Introducción.}

La historia de la justicia, los sistemas carcelarios y su impacto en la sociedad, ha sido tratada por numerosos autores, pero destaca la particular visión de Michael Foulcault (Foucault, 2000, pág. 60), al acuñar la sentencia "vigilar y castigar". La investigación a nivel de ensayo, sobre la temática de la vigilancia electrónica controlada y singularmente el empleo de las pulseras electrónicas en el control de reos y personas probadas de libertad va siendo cada vez más profusa en el mundo de las ciencias sociales.

El análisis de las condiciones de vigilancia y control en cualquier sociedad es para las instituciones gubernamentales y estatales un tema prioritario, condiciona acciones que el hombre desarrolla para enfrentar cualquier comportamiento indebido en la actuación social de los ciudadanos, que pueda acarrear hechos delictivos, esto claramente en un estado de derecho, va a encontrar conflictos con los llamados derechos universales y humano. En esta contradicción se sitúa la vigilancia controlada por dispositivos telemáticos en el caso de personas con privación de libertad o en espera de juicios en libertad provisional.

El objetivo fundamental discurre por entender a través de un análisis doctrinal y de derecho comparado, la evolución, aplicación y significado del monitoreo telemático y su transcendencia a nivel social en relación con los derechos humanos y la seguridad ciudadana. En este sentido es importante consignar que alrededor de este fenómeno se expresan implicaciones económicas, culturales, familiares, etc. Siendo una temática verdaderamente actual que concita un profundo debate en el seno de la sociedad moderna. 


\section{La seguridad ciudadana frente al derecho a la defensa en libertad de los procesados}

Vol. 3, núm. 4., (2019)

Félix Arquímides Valdez Rivera

Tanto a nivel de investigación, como de debate, la introducción de la monitorización electrónica de reos, o personas privadas de libertad, ha pasado en un inicio casi inadvertido, convirtiéndose luego en centro de noticias, reflexiones sociológicas y jurídicas, que a nuestro juicio no son todavía lo suficientemente fundamentadas y recogidas en el universo de las ciencias jurídicas. Algo que ha sido connotado, a partir de la mundialmente famosa fuga del narcotraficante, Joaquín "El Chapo" Guzmán de la cárcel de máxima seguridad del altiplano en México en el año 2015. Es por esta razón que se intentará dar una explicación general de la medida y desarrollar una evaluación crítica, tanto en términos jurídico como sociológicos, en relación con las percepciones sociales de los derechos humanos y la seguridad ciudadana.

Para tal objeto, se ha dividido la comunicación en tres partes, la primera, se propone ofrecer una evolución histórica de la medida, sus orígenes, señalando a los Estados Unidos, como país de origen del monitoreo telemático y sus tres fases de desarrollo, a saber, desde principios de la década de los 60 hasta mediados de los 70, desde mediados de los 70 hasta 1984 y, desde este año (fecha en la que se impuso por primera vez el uso del dispositivo) hasta la actualidad. Se abordan también de un modo breve la recepción de la medida en el continente europeo, revisando su aplicación en el Reino Unido, Portugal, Suecia, Suiza y España. Finalmente se expone la forma como se ha introducido en América Latina en esta problemática, destacando el hecho de que ello constituye una nueva forma de ver los procesos de control y vigilancia de las personas que pueden tener medidas sustitutivas de la privación de libertad.

La segunda parte se centra en la discusión sobre la aplicación del monitoreo telemático en el Ecuador y sus perspectivas. Así, se revisa el modo y ámbito de aplicación de la 


\section{La seguridad ciudadana frente al derecho a la defensa en libertad de los procesados}

Vol. 3, núm. 4., (2019)

Félix Arquímides Valdez Rivera

monitorización, De esta forma, al interior de esta nueva regulación, se valora como la "pulsera electrónica" cumple una función de complemento y refuerzo a dos nuevas instituciones sustitutas de las penas privativas de libertad: la reclusión parcial y la libertad vigilada intensiva.

La tercera parte se reflexiona a partir de la sociología del control y la economía política del castigo, desde las posturas epistemológicas de Michel Foucault y su idea de describir la monitorización como mecanismo de control estatal que actúa sobre el cuerpo del sujeto por medio de la vigilancia permanente. Además, se recurre a la teoría del control social y los campos culturales de Pierre Bourdieu, valorando como el control y normalización se ejerce con el fin de imponer un mensaje simbólico de vigilancia permanente a los sujetos (Romero Araque, 2016)

Finalmente, con Guilles Deleuze se postula la idea de que dicho mecanismo de vigilancia representa un dispositivo propio de las "sociedades de control", las que, en definitiva, se caracterizan por la diseminación de la vigilancia en la sociedad.

Además, es importante asumir los fundamentos teóricos enriquecedores que constituyen, los autores que de forma más perspicua han descrito la intervención penal y los mecanismos de control social por parte del Estado, en la actualidad, a saber: David Garland, Loic Wacquant y Alessandro de Giorgio (Garland, Wacquant, \& De Giorgi, 2006, pág. 35), el significado nos muestra una visión conceptual de la medida y su consolidación como una redefinición del papel del Estado, desde una concepción más ligada al bienestar, a una concepción vinculada directamente con la prevención, control, vigilancia y castigo. 


\section{La seguridad ciudadana frente al derecho a la defensa en libertad de los procesados}

Vol. 3, núm. 4., (2019) Félix Arquímides Valdez Rivera

Pero debemos de entender estos procedimientos deben estar acoplados a las características de los derechos humanos, para la Dra. Ligia Galvis, que las características de los derechos humanos se desprenden de la naturaleza múltiple de su concepción. "Desde el punto de vista filosófico, es características de los derechos humanos la pertenencia esencial a la persona inalienable e intransferible. Desde ese punto político, determinan la relación entre el individuo y el Estado y el modo de ser del sistema democrático. En este ámbito también podemos afirmar que los derechos humanos son inherentes a la democracia contemporánea. Desde el punto de vista jurídico, son normas de carácter positivo..." (Galvis Trujillo, 2008, pág. 64), ante ello es de suma importancia, que todo accionar de una democracia, tenga que ser un accionar bajo el respeto de los derechos humanos, con sus características fundamentales.

\section{2.- Monitoreo telemático judicial en Estados Unidos de Norteamérica}

Como ya hacíamos mención, el monitoreo telemático, o simplemente monitorización, surge en Estados Unidos en la década del 60. La doctrina ha identificado tres etapas en su evolución:

\section{Primera etapa:}

La primera fase de desarrollo de la medida se ubica entre principios de los años sesenta y mediados de la década de los setenta, recordar que esta es una época de luchas por los derechos civiles, nacimiento de la sociedad postmoderna y aparición de un incipiente y trepidante desarrollo de la telemática ${ }^{1}$. Esta etapa se destaca por la invención y desarrollo del mecanismo/

\footnotetext{
1 servicio de telecomunicaciones que permite la transmisión de datos informatizados a través del teléfono
} 


\section{La seguridad ciudadana frente al derecho a la defensa en libertad de los procesados}

Vol. 3, núm. 4., (2019)

Félix Arquímides Valdez Rivera

dispositivo (1964- 1970), gracias al trabajo de un grupo de psicólogos experimentados en Harvard, dirigidos por el Dr. Ralph Schwitzgebel. Estos científicos diseñaron un pequeño dispositivo llamado Bbehavior- Reinforce (BT-R), el que tenía por objeto "complementar la libertad condicional y la probación aplicadas a los sujetos reincidentes peligrosos", para Jenny Ardley (Ardley, 2005), en el principio y a pesar de su novedad, dicha medida no prospero en el sistema norteamericano.

\section{Segunda etapa:}

Esta segunda, se caracteriza por la aparición de diversos intentos tecnológicos, los cuales no cumplían los requisitos necesarios para ser efectivos, se sitúa entre medidores de control electrónico comunitario en el estado de Florida. Este mecanismo, no cautivo el interés por parte de los operadores del sistema, debido a sus problemas tecnológico, en efecto, era demasiado voluminoso, el campo de transmisión no supera un cuarto de milla y el campo de recepción era de, aproximadamente, dos millas. Lo cual lo sitúa en una especie de prototipo para posibilitar desarrollo tecnológicos futuros. Es importante consignar para el análisis que estas formas de control alcanzara un desarrollo ulterior ligadas a las personas y sus nuevas potenciales tecnológicas que trae la revolución visual y telemática.

\section{Tercera etapa:}

Esta fase del desarrollo se destaca por el resurgimiento del interés en el monitoreo telemático producto de la acción de un Juez en Alburquerque, Nuevo México, llamado Jack Love. Este Juez, en 1983, tras la propuesta de un experto en electrónica llamado Michael Goss, 


\section{La seguridad ciudadana frente al derecho a la defensa en libertad de los procesados}

Vol. 3, núm. 4., (2019)

Félix Arquímides Valdez Rivera

impuso la primera sentencia de arresto domiciliario con control electrónico. Dicho Juez, frustrado por la falta de alternativas al encarcelamiento, probo este mecanismo que se instalaba en el tobillo del sujeto. Posteriormente Love propuso al Departamento de Ejecución de Penas de Nuevo México la utilización de la monitorización para los sujetos condenados a prisión por conducir en estado de ebriedad y aquellos condenados a prisión por conducir en estado de ebriedad y aquellos condenados por delitos llamados "delitos de cuello blanco". Conjuntamente el National Institute of Justice (Instituto Nacional de Justicia de Estados Unidos de Norteamérica) aprecio la viabilidad de la tecnología y estableció su aplicación con el arresto domiciliario. Finalmente, en 1984 se adoptó en Palm Beach, Florida, el primer programa de control electrónico. Su establecimiento tenía por objeto reducir el hacinamiento en las cárceles (Romero Araque, 2016)

De este modo, comienza a ser aplicada la monitorización hasta nuestros días. El crecimiento exponencial en su aplicación resulta digno de destacar. En efecto, en 1988 había 2.227 condenados al control electrónico en diez estados del país del norte. Diez años después, en 1998, se cuentan alrededor de 95.000 personas sujetas a monitorización. En la actualidad, pese a la insuficiencia de datos, se calcula que hasta junio de 2009 solo en Palm Beach, Florida, existían cerca de 143.000 personas sujetas a la libertad condicional bajo monitoreo telemático ${ }^{2}$. Por otro lado, con otra muestra del alcance de la medida, desde el año 1989, en el pequeño condado de

${ }^{2}$ Cuadro estadístico No.- 1 


\section{La seguridad ciudadana frente al derecho a la defensa en libertad de los procesados}

Vol. 3, núm. 4., (2019)

Félix Arquímides Valdez Rivera

Cook, Illinois, se ha aplicado la vigilancia electrónica a más de 250.000 infractores. Esto demuestra la penetración del monitoreo telemático en el sistema penitenciario norteamericano ${ }^{3}$.

En cuanto al ámbito de aplicación, en la vigilancia electrónica en Estados Unidos existen importantes diferencias regulatorias entre los estados federales. En algunos estados, y como pena principal, se utiliza el monitoreo telemático junto al arresto domiciliario. Conjuntamente, también se utiliza como alternativa o forma alternativa de ejecución de la pena de prisión incondicional. En otros estados como medida adicional en el contexto de una suspensión condicional de la pena. Es por esta razón que se le considera entre las intermediate sanctions, es decir, se sitúa entre la ejecución de una pena y la suspensión condicional de la misma. No obstante lo anterior, en muchos estados se contempla el monitoreo telemático como una forma de vigilancia perpetua en contra de los sujetos que hayan sido condenados por crímenes de abuso y violencia sexual (luego de haberse cumplido la pena impuesta).

Respecto de los sujetos a quienes se puede imponer vigilancia electrónica, son destinatarios: personas en libertad condicional o en periodo de prueba, personas condenadas por delitos sexuales, inmigrantes esperando sentencia, individuos involucrados en caso de violencia doméstica, personas declaradas culpables de conducir en estado de ebriedad u otros delitos relacionados con el tránsito, aquellos acusados de delitos menores como allanamientos o violaciones a ordenanzas municipales, y personas jóvenes que enfrentan cargos ante Tribunales de Adolescentes.

\section{3.- El monitoreo y la vigilancia en Europa y América Latina}

${ }^{3}$ Cuadro estadístico No.-2 


\section{La seguridad ciudadana frente al derecho a la defensa en libertad de los procesados}

Vol. 3, núm. 4., (2019)

Félix Arquímides Valdez Rivera

En Europa, el monitoreo telemático se encuentra actualmente en aplicación por países tanto de la tradición anglosajona como de la tradición continental. Así, la monitorización se encuentra vigente, entre países, como Inglaterra, Gales, Francia, Portugal, Alemania, España, Suiza, Suecia e incluso fuera de Europa, en Australia (Oceanía).

El primero de estos países ha introducido, desde 1988, los primeros programas pilotos como una alternativa a la prisión preventiva y al hacinamiento carcelario (este llegaba a un total de 49.000 reclusos en el año 1987, 7.000 personas más de lo que el sistema podía soportar. El 20\% de ellos en prisión preventiva). Así, en 1991 se establece la Criminal Justice Act, ley que introduce la monitorización conjunta de los arrestos domiciliarios (sanciones comunitarias).

Paralelamente, en 1998, se establece la Crime and Disorder Act, por medio de la cual se introduce la aplicación del mecanismo para la libertad condicional (license condition). Posteriormente, en el año 2000, se dicta la Power of Criminal Courts (Sentencing Act). Este incorpora la exclusión orders, que son sanciones que involucran el alejamiento del condenado a ciertos lugares, en el contexto de sanciones de cumplimiento comunitario. Para terminar, en el año 2003 se dicta la Criminal Justice Act. Esta ley es la que actualmente rige en materia de monitorización y sanciones de cumplimiento comunitario. El aporte más relevante de esta ley son las extended and indeterminate sentences (penas extendidas e indeterminadas) a delincuentes sexuales y violentos de alto riesgo.

De forma conjunta, en relación con las tecnologías utilizadas, en el año 2001, se introduce el sistema biométrico (reconocimiento de voz) para el control del arresto domiciliario 


\section{La seguridad ciudadana frente al derecho a la defensa en libertad de los procesados}

Vol. 3, núm. 4., (2019)

Félix Arquímides Valdez Rivera

y, finalmente, entre 2004 y 2006 se introduce la tecnología GPS para el seguimiento de la libertad condicional.

Este sistema contempla la aplicación de la monitorización como apoyo a dos sanciones comunitarias, a saber: la Curfew Order (CO) y la Home Detention Ccurfew (HDC). La primera de ellas es una sanción comunitaria que puede ser reforzada con la vigilancia electrónica o con otrs sanciones comunitarias o pecuniarias. Por su parte, la segunda, permite abandonar anticipadamente la prisión (Romero Araque, 2016)

Estadísticamente, hasta el año 2011, se había monitoreado más de 700.000 casos y actualmente se monitorean cerca de 22.000. Esto convierte al Reino Unido en el programa de aplicación más extensiva, fuera de Estados Unidos. En Francia, las medidas que permiten la vigilancia electrónica fueron introducidas en un periodo de tres años; la primera de las leyes fue la No. 2005-1549, la que permite la aplicación de un seguimiento continuado mediante dispositivos electrónicos a sujetos que representan un alto riesgo de reincidencia tras el cumplimiento de la sanción impuesta. Asi, se permite la aplicación de la vigilancia electrónica al adulto condenado a una pena privativa de libertad igual o superior a siete años. Esta sanción permite determinar la localización espacial del sujeto por un periodo de dos años, prorrogables por otros dos periodos (dependiendo si la infracción es crimen o un delito simple, según la legislación interna).

En segundo lugar, la ley del 25 de febrero de 2008, introduce surveillance de sureté (vigilancia de seguridad). Esta medida postpenitenciaria permite la vigilancia continuada del sujeto que ha sido condenado a una pena igual o superior a quince años de prisión. Bajo dicha 


\section{La seguridad ciudadana frente al derecho a la defensa en libertad de los procesados}

Vol. 3, núm. 4., (2019)

Félix Arquímides Valdez Rivera

vigilancia, el condenado debe cumplir determinadas obligaciones, entre las cuales se encuentra someterse a un régimen de vigilancia electrónica.

Finalmente, la Ley No. 2010-242, del 10 de marzo de 2010, vino a confirmar el papel de la vigilancia electrónica. Esta ley, en su artículo 5, establece que si bien el condenado puede elegir la utilización de la vigilancia electrónica, este es advertido que de no aceptar dicho dispositivo o de incumplir las obligaciones, de aceptarlo, el sujeto puede ser internado en un centro socio- médico- judicial.

En Suiza, la vigilancia electrónica ha sido fácilmente aceptada en algunos cantones por ejemplo Ginebra, Basilea y Vaud, cuyas regulaciones no son idénticas, pero fundamentalmente, rechazadas en otros como por ejemplo Zurich, por lo que solo se encuentra regulada a nivel local. En estos cantones se utiliza el monitoreo telemático como una forma de supervisión del arresto domiciliario por no más de doce meses.

En Australia, es posible diferencias la aplicación de la vigilancia electrónica en tres fases: En primer lugar, previo a la sentencia (de uso provisional), como parte de la concesión de la libertad bajo fianza, para quienes solicitan asilo mientras sus solicitudes se están procesando y, finalmente, se utiliza cuando se dictan ordenes de restricción de acercarse a la víctima. En segundo lugar, al momento de la sentencia, el monitoreo electrónico puede imponerse bajo ciertas restricciones de libertad que se imponen al condenado y como una forma de prevención de acercamiento a la víctima del delito por el cual es condenando. Finalmente, se puede imponer luego de haber cumplido con la pena prisión, cuando se produce una liberación anticipada del recluso (Romero Araque, 2016) 


\section{La seguridad ciudadana frente al derecho a la defensa en libertad de los procesados}

Vol. 3, núm. 4., (2019)

Félix Arquímides Valdez Rivera

Portugal, por otro lado, ha establecido el sistema de vigilancia electrónica como mecanismo para controlar el arresto domiciliario de imputados y condenados, el seguimiento de la libertad condicional y los delitos relacionados con la violencia intrafamiliar tanto para condenados como para imputados.

En Alemania, la entrada en vigor el 1 de enero del 2011 de la Gesetz zur Neuordnung des Rechts der Sicherungsverwarhrung und zu begleitenden Regelungen, aprobada el 22 de diciembre de 2010, permitió la vigilancia permanente sobre sujetos por medio de mecanismos electrónicos. No obstante, el monitoreo electrónico ya era ocupado en el ámbito de medidas cautelares y de la probation, es decir, siempre en forma de controlar la permanencia o ausencia del sujeto en su domicilio, utilizando para ello sistemas estáticos de radiofrecuencia.

La introducción en el sistema jurídico alemán de la vigilancia continuada por medio del sistema GPS se debe a la sentencia del Tribunal Europea de Derechos Humanos, del 17 de diciembre de 2009, el cual fallo en contra de la aplicación retroactiva de la Sicherungsverwahrung (custodia de seguridad). Esta medida post sancionatoria permitía la internación de delincuentes sexuales y considerados peligrosos con posterioridad al cumplimiento de la pena de prisión. Dicha resolución del tribunal permitió la liberación de cientos de sujetos considerados peligrosos. De este modo, se estableció la posibilidad de un seguimiento continuado mediante un dispositivo GPS.

Las hipótesis en las que se aplica la monitorización son dos: En primer lugar, a los sujetos que hayan finalizado la ejecución de una pena de prisión superior a tres años en delitos contra la vida, integridad física, libertad y libertad sexual. En segundo lugar, se aplica a los sujetos que 


\section{La seguridad ciudadana frente al derecho a la defensa en libertad de los procesados}

Vol. 3, núm. 4., (2019)

Félix Arquímides Valdez Rivera

han terminado de cumplir una medida de seguridad a los que previamente se les aplicaba la custodia de seguridad.

En Suecia, por su parte, es uno de los países en Europa que ha utilizado hace más años el monitoreo telemático (puesto a prueba en 1994 y oficialmente utilizado desde 1996). En cuanto a su ámbito de aplicación, la vigilancia electrónica se aplica en los siguientes casos: en primer lugar, es utilizado como una forma de controlar las condiciones impuestas a un sujeto a que remite la pena prisión de corta duración (especialmente, pero no exclusivamente, se dirige a quienes resulten condenados por conducir en estado de ebriedad). En segundo lugar, se utiliza en los casos de libertad anticipada. Existen, a su vez, proyectos de aplicación en materia de delincuencia juvenil (personas de 15 a 18 años de edad) y órdenes de alejamiento de víctimas (Romero Araque, 2016)

Finalmente, en España se ha introducido, en primer lugar, mediante el Reglamento Penitenciario de 1996. El Reglamento establece, en su artículo 86.4, una nueva forma de cumplimiento del régimen abierto que permite a los internos de tercer grado sometidos a los regímenes de semi libertad, sustituir la permanencia en el centro penitenciario con vigilancia electrónica durante ocho horas, o pernoctar en el mismo.

En segundo lugar, también se establece en el ámbito de la violencia doméstica. En el año 2003 la LO 15/ 2003 introduce en el país ibérico la posibilidad de aplicar la monitorización como medio de control junto a la pena de alejamiento del artículo 48 del Código Penal Español (por medio de la pena de privación de derecho de residir o acudir a un lugar determinado). Finalmente, mediante la misma ley, también puede imponerse la vigilancia electrónica como un 


\section{La seguridad ciudadana frente al derecho a la defensa en libertad de los procesados}

Vol. 3, núm. 4., (2019)

Félix Arquímides Valdez Rivera

mecanismo de control de la pena llamada localización permanente, reglada en los artículos 33 al 35 del Código Penal Español, solamente aplicada a las infracciones constitutivas de falta leve.

En cuanto a América Latina, el panorama es similar. En efecto, se ha avanzado en la adopción de tecnologías de control a distancia en países como Argentina, Colombia, Panamá y Uruguay.

En Argentina, la provincia de Buenos Aires avanzó en su aplicación mediante un plan piloto desde 1997. El dispositivo utilizado se trataba de un anillo ubicado en la muñeca o en el tobillo, que permitía cautelar al imputado mientras el proceso judicial aún tenía lugar, es decir, durante el tiempo en que el sujeto esperaba su condena.

Dicha finalidad fue atribuida, fundamentalmente, para combatir el hacinamiento carcelario, toda vez que cerca del $85 \%$ de la población privada de libertad se encontraba en dicha condición a causa de esta medida cautelar. El principal problema era su escasa cobertura, pues solo era posible aplicar el mecanismo a trescientas personas. En la actualidad, dicho programa piloto fue cerrado, por lo que este país no cuenta con monitoreo telemático.

Por su parte, en Colombia se estableció formalmente el monitoreo telemático según la Ley No. 1142, desde el año 2007. Su implementación se entregó a un reglamento mediante el Decreto No. 177 en el año 2008, el que establece los requisitos para imponer la vigilancia electrónica. Dicho sistema de monitorización.

Es utilizado como medida sustitutiva a la pena de prisión y su aplicación la determina el juez de ejecución de las personas a quienes hayan sido sancionados por delitos de escasa y 


\section{La seguridad ciudadana frente al derecho a la defensa en libertad de los procesados}

Vol. 3, núm. 4., (2019)

Félix Arquímides Valdez Rivera

mediana gravedad. El año 2010 se produce su implementación definitiva a todo el territorio del país, contempla, a su vez, las tecnologías de GPS pasivo, GPS activo y reconocimiento de voz.

En Panamá, mediante la resolución No. 46 emitida por el Ministerio Público, el 29 de diciembre del año 2009, se introduce permanentemente el uso de brazalete electrónico para garantizar el cumplimiento de las medidas cautelares. Tal mecanismo se plantea como un sistema de seguimiento permanente y se encuentra bajo la supervisión de la Dirección de Asistencia y Coordinación Penitenciaria de la Procuraduría General de la Nación.

En el caso de Uruguay, la Corte Suprema de Justicia habilito el uso de estos sistemas en noviembre del año 2003, solo para los imputados primarios por delitos leves, hurtos pequeños, homicidios culposos en accidentes de tránsito, y solo por medio de resolución judicial. Actualmente el Ministerio del Interior de encuentra en proceso de licitación de la compra o alquiler de pulseras electrónicas para presos que sean enviados a prisión domiciliaria.

\subsection{Valoración crítica de la evolución histórica abordada, sobre el sistema de monitoreo electrónico}

La historia de la introducción de este tipo de medidas, y sus implicaciones en el orden social, los derechos humanos y la seguridad ciudadana en diversos países permiten considerar ciertas características generales relativas a la utilización de la monitorización, finalidad, tecnologías ocupadas, procedimientos de aplicación, contexto de introducción y beneficios propios de la medida, destacando que: 


\section{La seguridad ciudadana frente al derecho a la defensa en libertad de los procesados}

Vol. 3, núm. 4., (2019)

Félix Arquímides Valdez Rivera

- Respecto del uso del monitoreo telemático, cabe mencionar que constituye un mecanismo de vigilancia que se instala en el cuerpo del sujeto (en la mayoría de los casos), con el objeto de verificar la ubicación espacio- temporal del mismo, De este modo es posible controlar el cumplimiento de las distintas medidas, a saber, medidas cautelares, penas sustitutivas y, finalmente la pena de prisión.

- Las tecnologías utilizadas han sido tres: monitorización por radiofrecuencia, dispositivo no removible que se instala en el tobillo del sujeto. Este dispositivo envía señales a una unidad básica instalada en el domicilio de la persona, el que a su vez envía información al centro de monitoreo. En segundo lugar, se encuentra el monitoreo biométrico, mecanismo de reconocimiento de voz, instalado en una computadora, que tiene por función llamar aleatoriamente al domicilio del sujeto con el fin de verificar la presencia del mismo, finalmente, se utiliza el monitoreo satelital o GPS, mediante el cual se sigue al sujeto a todos los lugares donde se dirige por medio de la emisión de señales satelitales al centro de monitoreo, En este último caso, la extracción de los datos es ilimitada. Constituye un mecanismo ideal para el cumplimiento de las zonas de exclusión.

- Dentro de los procedimientos de aplicación, cabe consignar que todos los países comenzaron la aplicación por medio de programas piloto, otros, por su parte, ya han alcanzado la aplicación completa de la medida (Estados Unidos de Norteamérica, Reino Unido, Panamá, Colombia). En este sentido es importante valorar como en algunos países de Latinoamérica a pesar de la real diferencia tecnológica, con el mundo desarrollado ya se cuenta con estas tecnologías. 


\section{La seguridad ciudadana frente al derecho a la defensa en libertad de los procesados}

Vol. 3, núm. 4., (2019)

Félix Arquímides Valdez Rivera

- En relación con la finalidad de la medida, la mayoría de los países que han instalado la vigilancia electrónica lo han hecho como respuesta al creciente hacinamiento en las cárceles. A esto se suma la posibilidad de mantener el control sobre los sujetos a un menor costo. En efecto, se estima que en Estados Unidos e costo de la monitorización es seis veces menor que la prisión. De este modo, el ahorro en la construcción de nuevas cárceles es asumido por este nuevo mecanismo como una virtud que motiva su utilización.

- El contexto dentro de cual se aplica la medida permite sostener la existencia de una diferencia entre países de tradición anglosajona y aquellos de tradición continental. Así, los primeros no han implementado el sistema por medio de una regulación rigurosa y pormenorizada, sino que lo han establecido como una atribución judicial al dictar sentencia. Su regulación queda sujeta a las disposiciones del servicio penitenciario respectivo. Por su parte, en los países de tradición continental existen normas y leyes que introducen la medida y reglamentos administrativos que regulan su aplicación.

- Producto del carácter permanente de la vigilancia que impone, se ha llegado a estimar que uno de sus mayores beneficios sería la eficacia en la normalización de la conducta. Se ha estimado que permitiría reducir la reincidencia y, por tanto, potenciar la reinserción en la comunidad. El problema de esta afirmación reside en que, como sostiene la mayoría de la doctrina en la materia, ninguno de los estudios que aborda la efectividad de la medida resulta convincente, especialmente por los errores metodológicos cometidos en su elaboración. La única excepción a este respecto es el 


\section{La seguridad ciudadana frente al derecho a la defensa en libertad de los procesados}

Vol. 3, núm. 4., (2019)

Félix Arquímides Valdez Rivera

caso de los delincuentes sexuales, sobre quienes existe un estudio que establece la disminución de las infracciones a la ley con posterioridad a la aplicación del monitoreo, Sin embargo, en esta clase de delincuentes solo resulta efectivo para aquellos de más alto riesgo ${ }^{4}$. En relación con los delincuentes juveniles, se ha destacado que dicho mecanismo de vigilancia puede provocar un aumento en su comportamiento delictivo. Previo al análisis de la medida en nuestro país, cabe resaltar el supuesto efecto humanizador que se le atribuye a la medida. Sobre este respecto nos pronunciamos en los sujetos se ubiquen fuera del alcance de la prisión, la medida esconde efectos y finalidades ulteriores relacionadas con la extensión del control y vigilancia estatal sobre la sociedad.

En este punto del análisis es importante consignar como la paulatina introducción de este tipo de medidas ha ido acarreando una opinión pública que no siempre es favorable y que ve en este mecanismo una doble amenaza, por una parte, la reincidencia delincuencial y por otra las posibilidades del surgimiento de un excesivo control estatal sobre la sociedad.

Aquí aparece una vieja razón social, y modos de interpretación de la historia y la justicia en relación con la rehabilitación social de los seres humanos que han delinquido. Se observa como la presión social ante estas medidas de cuenta de temores y formas tradicionales de ver la prisión y la cárcel como lugar de freno para delincuentes al propio tiempo que todavía la sociedad no concibe en su totalidad el modo en el que puedan construirse espacios de prevención y control social desde las potencialidades tecnológicas actuales.

\footnotetext{
${ }^{4}$ Según una concepción actuarialista
} 


\section{La seguridad ciudadana frente al derecho a la defensa en libertad de los procesados}

Vol. 3, núm. 4., (2019)

Félix Arquímides Valdez Rivera

\section{4.- Ecuador: EI Monitoreo y la vigilancia electrónica}

La Constitución declara un extenso catálogo de derechos destinados a proteger la dignidad de las personas, la integridad de las distintas colectividades titulares de derechos y de la naturaleza. Para dotarles a los derechos de efectividad, por una parte, establece un vínculo directo entre los derechos y la organización del Estado y, por otra, diseña un sistema amplio de garantías genéricas como la supremacía de la Constitución, la reserva de ley, el procedimiento agravado para su forma, etc., y garantías específicas como las garantías normativas, las garantías institucionales o de políticas públicas y las garantías jurisdiccionales. Para reforzar el sistema garantista, establece un conjunto de principios de aplicación de los derechos, que vincula a todo el Estado, sus instituciones, órganos de poder público, instancias, así como a los particulares (Chávez Vallejo, 2013, pág. 34)

En el Ecuador, el uso de dispositivos electrónicos fue introducido de manera reciente en la legislación como un instrumento de control y fiscalización de las medidas cautelares como formas de ejecución de la prisión preventiva, es decir, como una verdadera ayuda a otras medidas cautelares como por ejemplo el arresto domiciliario, la detención y la medida extraordinaria de la prisión preventiva.

Pero, en legislación comparada y como lo hemos analizado el dispositivo de vigilancia electrónica, se considera un instrumento de control aplicado de forma amplia en la ejecución de una pena y como medida cautelar de carácter autónoma, el monitoreo electrónico, como se expuso, ha sido concebido como modelo de ejecución de la prisión preventiva y provisoria; una verdadera alternativa a los regímenes de privación de libertad de los centro carcelarios, sirviendo 


\section{La seguridad ciudadana frente al derecho a la defensa en libertad de los procesados}

Vol. 3, núm. 4., (2019)

Félix Arquímides Valdez Rivera

de garantía a los regímenes abiertos, semi- abiertos e incluso como una garantía de la prisión preventiva.

La matriz constitucional, dispone que la privación de la libertad es una medida excepcional, en donde prima la justicia restaurativa, como modelo de justicia en materia penal que ha venido a reemplazar a la justicia retributiva o punitiva, pero aún no se aplica tal como consta en la normativa sobre la materia, prevaleciendo aun en nuestro sistema la justicia retributiva que se basa sola y únicamente en la sanción penal, de forma esencial la pena privativa de libertad, sanción que se cumple en los Centro Penitenciarios del país, en donde se ejecuta el sistema de rehabilitación, que poco a poco están cambiando para dejar atrás el hecho bochornosos de hacinamiento y de corrupción que por años los centros ecuatorianos, han tenido, centro carcelarios considerados por el pueblo como Universidades del Delito.

Como sabemos en Ecuador, desde el año 2008, se transforma en un Estado garantista, Gina Chávez sostiene que "El Estado garantista no es el resultado de la aplicación de una moda. Es la respuesta lógica para paliar las consecuencias de la crisis del modelo neoliberal que impero en el país durante los últimos veinticinco años" (Chávez Vallejo, 2013, pág. 46)

Las reformas a los artículos 548 y 549 del Código Orgánico Integral Penal, de ahora en adelante COIP, dichas reformas consisten en reglamentar de forma adecuada la aplicación de estos dispositivos, cuya finalidad es subsanar las gravísimas omisiones que existen en nuestra legislación, beneficiando para ello a las personas cuya imposición de pena privativa de libertad sea menor a los cinco años. 


\section{La seguridad ciudadana frente al derecho a la defensa en libertad de los procesados}

Vol. 3, núm. 4., (2019)

Félix Arquímides Valdez Rivera

Se hace una amplia referencia en nuestro país, sobre los principios y derechos que el propio Estado debe de cumplir, ya que en las escasísimas oportunidades en que se permiten la utilización de los dispositivos electrónicos de geoposicionamiento, estos en la práctica aun no son aplicados a un ciento por ciento, por lo que fracasa en cierta medida los objetivos por los cuales fue planificado, conculcando expresamente la norma legal por falta de recursos y del compromiso social para con las personas privadas de libertad, lo que realmente no es de sorprender en nuestro país, a pesar del discurso del gobierno que enaltece la dignidad del ser humano, en base de los preceptos socialistas, pero la realidad que la tónica del gobierno hace gala del más retorico populismo, al puro estilo velasquista.

En el artículo 195 de la Constitución de la República del Ecuador, en donde consta el principio de mínima intervención penal, principio que el jurista italiano Dr. Luiggi Ferrajolí, expresa lo siguiente: "Derecho penal mínimo significa la reducción al mínimo de las circunstancias penales y su codificación general mediante la despenalización de todas aquellas conductas que no ofendan bienes fundamentales y que saturan el trabajo judicial con un dispendio inútil e inocuo de aquel recurso escaso y costoso que es la pena y tienen el triple del debilitamiento general de las garantías, de la ineficacia de la maquinaria judicial y de la devaluación de los bienes jurídicos merecedores de tutela penal” (Ferrajolí, 1995, pág. 113)

Pero en nuestro país la realidad es muy distinta, ya que es vox populi que la Policía Nacional, por intermedio de sus voceros al ser preguntada por distintos medios de comunicación sobre la aplicación de la vigilancia electrónica, responden que no cuentan con el personal, para poder ejecutar la vigilancia de esta medida, ante esta frecuente "falta de personal", le ha valido a 


\section{La seguridad ciudadana frente al derecho a la defensa en libertad de los procesados}

Vol. 3, núm. 4., (2019)

Félix Arquímides Valdez Rivera

Ecuador ser condenado por la Corte Interamericana de Derechos Humanos, ya que nuestro país ha hecho caso omiso a estas reiterativas conductas por parte de entidades encargadas de precautelar derechos de todas las personas, se agrega ahora la casi también recurrente frase "falta de equipos electrónicos de vigilancia personal”, lo que a resumidas, vulnera el principio del derecho penal mínimo y de una justicia garantista, lo que obviamente permite sustentar sin reparo alguno, que en Ecuador, ante la falta de medios y de compromiso, existe una justicia retributiva o punitiva de forma paralela con un derecho penal del enemigo.

Ante ello, es necesario citar lo que expone el tratadista Víctor Goncalvez Machado, en su libro titulado El fracaso de la pena de prisión: alternativas y soluciones, señala acertadamente: "La prisión es una institución antiliberal, desigual atípica, extralegal y extrajudicial, que pervierte, corrompe, deforma, envilece y embrutece, siendo una sucursal del infierno, drásticamente lesiva para la dignidad del ser humano, penosa e inútilmente aflictiva, considerada, además, una verdadera fábrica de reincidencia y una industria del crimen" (Goncalvez Machado, 2009, pág. 109)

No se determina con claridad en la nueva Ley Penal ecuatoriana, que la vigilancia electrónica personal constituya una pena autónoma, que debe de aplicarse a los delitos leves, siendo y como debe de ser, la privación de la libertad de ultima ratio, lo que es aplicar en plenitud el derecho penal mínimo, dando posibilidad al infractor, encontrarse en su entorno libre y controlado electrónicamente, para poder rehabilitarse con prontitud y mayor eficacia.

La Dra. María Poza Cisnero, considera: "La vigilancia electrónica es un método de control y observación que se puede aplicar tanto a seres humanos como a cosas, a fin de 


\section{La seguridad ciudadana frente al derecho a la defensa en libertad de los procesados}

Vol. 3, núm. 4., (2019)

Félix Arquímides Valdez Rivera

conocer la ubicación exacta, la vía y el desplazamiento del objeto supervisado (...) consiste en el método que permite controlar donde se encuentra, el alojamiento o aproximación respecto de un lugar determinado de una persona o cosa" (Poza Cisnero, 2001, pág. 61)

De forma general el sistema de vigilancia electrónica ofrece, a pesar de sus limitaciones, que la persona a quien se le impone este tipo de medida tiene una vida personal absolutamente normal, en razón de que no lo aparte de su núcleo familiar, de su entorno educativo, laboral, ya que goza de su libertad pero vigilada, esta representa un avance tecnológico de suma importancia judicial, social y científica, puesto que ofrece fiscalizar el irrestricto cumplimiento de las medidas judiciales que mediante sentencia motivada hayan sido impuestas a determinada persona por haber adecuado su conducta a un injusto penal previamente establecido en la ley punitiva, siendo esta medida impuesta por el Juzgador, debiendo establecer un estricto programa de cumplimiento, que se puede cumplir en su residencia, como si fuera la prisión domiciliaria que sirve de ejemplo focalizado, ya que desde la óptica técnica la vigilancia electrónica consiste en dispositivos con resistencia al agua y antialérgicos, como tobilleras o muñequeras.

El excelso jurista Abel Téllez Aguilar, define al sistema de vigilancia electrónica como: "Los medios telemáticos de control de penas consistentes en el empleo de transmisión de información a través de medios telefónicos o telemáticos con el objeto de conocer de forma continuada o puntual la ubicación espacial de los sujetos sometidos al control, y, por lo tanto, verificar su sujeción al cumplimiento de la pena” (Téllez Aguilar, 2011, pág. 102)

La favorabilidad de la aplicación de los dispositivos de vigilancia electrónica, se los cataloga de beneficioso, sin que esto de a entender que se aplique teorías abolicionistas de la 


\section{La seguridad ciudadana frente al derecho a la defensa en libertad de los procesados}

Vol. 3, núm. 4., (2019)

Félix Arquímides Valdez Rivera

pena, tal como lo señala el tratadista español Dr. Fausto Gudín Rodríguez Magariños, quien expresa: "La opción que nos brinda la cárcel electrónica, como alternativa a la cárcel tradicional, es que nos permite salir del circulo vicioso que supone recluir, castigar y marginalizar y esperar que acaezca algo positivo. La cárcel electrónica no implica liberar al individuo de la sombra del Estado, este sigue ahí y por tanto la libertad igualmente se ha perdido, decir lo contrario sería falsear los hechos. Pero si nos permite dar una utilidad a la reclusión, a la vez que podemos observar si posteriormente el individuo puede o no reencausar su vida, nos permite ser más humanos pues está con los suyos y poder estudiar, trabajar y tener la ilusión de que algún día demostrando que no es lo que fue, que puede vivir plenamente en sociedad" (Rodríguez Magariños, 2004-2005, pág. 85), como se puede analizar el penalista español, muestra su postura favorable a la aplicación del sistema de monitoreo electrónico, ya que se comparte el criterio que la cárcel no es precisamente un centro de rehabilitación, como se había apreciado en la legislación comparada, que incluso en casos que revisten mayor gravedad, cuando el condenado a cumplido un tercio de la pena impuesta en su sentencia condenatoria, si ha mantenido buena conducta, se le otorga este beneficio como en la República de Chile, conocido como el régimen mixto.

El vocero del Servicio de Vigilancia Electrónica de la República de Portugal, Dr. Nuno Caiado, al referirse a ciertos sectores que se oponían a la implementación del sistema de vigilancia electrónica, señala lo siguiente: "En sus formas más comunes, la Vigilancia Electrónica incide en primer lugar en el cuerpo, sobre su permanencia en un local o sobre su posición en el espacio público. En esta materia importa comprender que el condicionamiento del 


\section{La seguridad ciudadana frente al derecho a la defensa en libertad de los procesados}

Vol. 3, núm. 4., (2019)

Félix Arquímides Valdez Rivera

cuerpo no significa necesariamente o automáticamente, la modificación de la motivación y del comportamiento criminal. Sin embargo, puede en ciertos casos contribuir. La verdad, ese cambio -que debería ser el objeto escatológico de la pena- solo podrá ser alcanzado a través de la combinación de la voluntad del delincuente, de las formas de control y de las formas de acompañamiento que incluyen módulos de trabajo social que interfieran en el comportamiento criminal. Por otro lado, el miedo del toque corporal puede deberse a miedos ancestrales relacionados con la aplicación de malos tratos físicos o el uso de grilletes, expresión que aún no desapareció del léxico corriente, puesto que sobrevive, a pesar de haberse creado un versión moderna/ electrónica ("grillete electrónico" o "esposa electrónica"), en un discurso centrado en la idea de inmovilización. La asociación de grilletes o esposas con los dispositivos electrónicos es totalmente incorrecta o ilegitima. Aquello se basa en el desconocimiento de la naturaleza de la Vigilancia Electrónica, toda vez que el grillete era un instrumento de incapacitación mecánica y física del cuerpo de una persona condenada, orientado a impedir de modo forzado su movilidad. Los dispositivos electrónicos de la Vigilancia Electrónica, pese a que se encuentran sobre el cuerpo, no implican un impedimento físico para su movilidad, por lo tanto no pueden impedir por si mismos que el cuerpo permanezca en un local o que se salga de un confinamiento obligatorio" (Caiado, 2013, pág. 15)

En países como Colombia, la Corte Constitucional, mediante sentencia C-185/11, señalo expresamente cómo funcionan los sistemas de vigilancia electrónica, resolviendo de la siguiente manera: "El legislador se encargó de regular la forma en que debía funcionar la vigilancia electrónica, para lo cual desarrollo tres modalidades de vigilancia electrónica, las cuales debe 


\section{La seguridad ciudadana frente al derecho a la defensa en libertad de los procesados}

Vol. 3, núm. 4., (2019)

Félix Arquímides Valdez Rivera

ser asignadas por la autoridad judicial competente de acuerdo con la disponibilidad de las mismas y las fases previstas para ser implementadas. i. El seguimiento pasivo RF: Es el sistema de vigilancia electrónica a través del cual se instala un dispositivo consistente en un brazalete o una tobillera en el cuerpo del condenado, sindicado, imputado o acusado, el cual trasmite a una unidad receptora, la que a su vez se encuentra conectada a una línea telefónica convencional. ii. El seguimiento activo- GPS. Es el sistema de vigilancia electrónica a través del cual se instala un dispositivo consistente en un brazalete o tobillera en el cuerpo del sindicado, imputado o acusado, el cual llevara incorporado una unidad GPS (Sistema de posicionamiento global), la cual transmitirá la ubicación del beneficiario, indicando si ha llegado a zonas de exclusión. La norma establece que cuando el beneficiario del dispositivo llegue al lugar establecido para el cumplimiento de la medida de aseguramiento, la información que así lo indique será transmitida al centro de monitoreo vía telefónica o móvil, sin que durante el transcurso del día se haya perdido la transmisión inherente al sistema de vigilancia electrónica. iii. El reconocimiento de voz. Es el sistema de vigilancia electrónica a través del cual se lleva a cabo una llamada a un lugar de residencia del condenado o sindicado, y autentica su identidad comparando su voz contra una impresión de voz previa tomada durante el proceso de registro." (Función del Sistema de Vigilancia Eelectronica en Colombia, 2011)

La pena privativa de libertad ha demostrado no tener efectos rehabilitadores, porque esta representa el ejercicio del jus puniendi estatal que no es más que el resultado de una justicia retributiva a la que le interesa solo sancionar al infractor de la ley, ya que los Centros de Rehabilitación Social al no cumplir con las Reglas Mínimas de Tratamiento de la ONU, las 


\section{La seguridad ciudadana frente al derecho a la defensa en libertad de los \\ procesados}

Vol. 3, núm. 4., (2019)

Félix Arquímides Valdez Rivera

personas privadas de la libertad viven en condiciones que atentan contra sus derechos humanos (Romero Araque, 2016, pág. 91)

Como se había dicho, en Ecuador, la vigilancia electrónica se encuentra inserta en el Código Orgánico Integral Penal:

- El numeral 4 del artículo 522 del COIP, hace referencia a los dispositivos de vigilancia electrónica como medida cautelar, a fin de asegurar la presencia del procesado;

- Los numerales 2 y 3 del artículo 558 del COIP, la vigilancia electrónica es aplicable a la persona procesada para que no se acerque a la víctima, testigos y determinadas personas, en cualquier lugar donde se encuentren y la prohibición al procesado o procesada de efectuar actos de intimidación o persecución a la víctima o a miembros del núcleo familiar por sí mismo o por intermedio de terceras personas, medidas amparadas al tenor de lo dispuesto en el inciso $1^{\circ}$ del artículo 559 Ibídem.

- En el antes citado artículo 559, en su inciso $2^{\circ}$, indica que también los dispositivos de vigilancia electrónica pueden ser utilizados por la víctima, testigos u otra persona que participe en el proceso penal.

- En cuanto a los regímenes de rehabilitación social, semi abiertos contemplados en el artículo 698 de la ley penal vigente, y el régimen abierto constante en el artículo 699 Ibídem, que facultan al Juez o Jueza de Garantías Penitenciarias para ordenar la vigilancia electrónica por medios de los dispositivos detallados en la presente. 


\section{La seguridad ciudadana frente al derecho a la defensa en libertad de los procesados}

Vol. 3, núm. 4., (2019)

Félix Arquímides Valdez Rivera

Como se observa en el COIP, constan enunciados normativos pertinentes a la vigilancia electrónica en Ecuador, pero no existe una norma específica que ofrezca parámetros procedimentales de estos dispositivos, a pesar de la normativa citada, lo que hemos venido sosteniendo es la falta de dichos dispositivos y del compromiso de las entidades regentes y gubernamentales.

En fecha 26 de enero del 2016, en el periódico virtual www.ecuadorinmediato.com, en su informe periodístico titulado Desde este mes se probarán brazaletes electrónicos para PPL $^{5}$, con lo que se evidencia que antes de la fecha de la nota periodística, no se ha podido implementar la medida por falta de recursos (Ecuador inmediato, 2016).

Desalentadora noticia para quienes desean beneficiarse de este mecanismo electrónico, pero, en el diario El Universo de fecha 21 de marzo del 2016, bajo el titulo Brazalete electrónico se utilizara en cuatro fases de pre libertad, se recoge lo expuesto por la Ministra de Justicia, Lady Zuñiga, quien expuso lo siguiente: "aquellas personas que están siendo procesadas o investigadas por delitos legales, es decir, a quienes en estos momentos se está siguiendo un juicio y que seguramente pueden estar ya dentro del sistema penitenciario, serían las beneficiarias", siguiendo con la nota periodística "Ocho empresas participan en la licitación del dispositivo que se utilizará, además, en el caso de otorgar medidas de protección a víctimas de violencia intrafamiliar, para evitar que el agresor se acerque; o a quienes por causas especiales, como atención médica o embarazos, no puedan estar dentro de un centro carcelario. Además, en

\footnotetext{
${ }^{5}$ PPL son las siglas de personas privadas de su libertad
} 


\section{La seguridad ciudadana frente al derecho a la defensa en libertad de los procesados}

Vol. 3, núm. 4., (2019)

Félix Arquímides Valdez Rivera

los casos que el Juez dictamine que no es necesario el cumplimiento de una pena con cárcel sino que se pueda utilizar uno de los dispositivos electrónicos..." (Diario El Universo, 2016).

Como todo proceso, siempre conlleva demoras o retardos en la adquisición por intermedio del portal de compras públicas, pero el Ministerio de Justicia, Derechos Humanos y Cultos, en una publicación al medio de comunicación vía internet ecuadorinmediato.com, indican que desde este mes (septiembre) se probaran brazaletes electrónicos para PPL, cuya reseña es la siguiente: "Desde este mes se realizarán las primeras pruebas del brazalete electrónico para las personas privadas de la libertad (PPL). El uso de este dispositivo se implementará para reducir la población carcelaria. Esta alternativa de la prisión preventiva y considerada como una medida sustitutiva efectiva, no solo para descongestionar cárceles, sino, pretendiendo la reinserción social de los condenados. El dispositivo de vigilancia electrónica se utiliza en el mundo desde 1980 con buenos resultados para el control de la población carcelaria. Es la medida alternativa para los privados de libertad por delitos menores o para quienes enfrentan prisión domiciliaria, lo cual reduce notablemente el costo y el riesgo de violar la custodia policial..." (ecuadorinmediato, 2016).

Como se puede observar ya una vez adquiridos los brazaletes electrónicos, el Ministerio de Justicia, está empezando a con su implementación, capacitando al personal dependiente del Ministerio para su optima ejecución, a esto se suma comentarios de varios altos funcionarios como el Defensor Público, Dr. Ernesto Pazmiño, quien indica al respecto de la implementación del brazalete electrónico, lo siguiente: "Esto, para que el Juez y la sociedad estén tranquilos de 


\section{La seguridad ciudadana frente al derecho a la defensa en libertad de los procesados}

Vol. 3, núm. 4., (2019)

Félix Arquímides Valdez Rivera

que esta persona no va a fugarse y va a estar presente en la audiencia de juicio" (Ecuador inmediato, 2016).

En Ecuador, desde que se constitucionalizo el derecho penal, empezó a dar fin el viejo marco de rehabilitación penitenciario, para dar inicio a la verdadera humanización del derecho penal, obligando a todos los actores que son parte de este ámbito a dar más énfasis a la verdadera y real rehabilitación, cuya finalidad es de reinsertar un sujeto que pago su pena a la sociedad, como una segunda oportunidad de vida.

Ya no siendo la pena privativa de libertad como lo hemos expuesto, una regla general, que el juzgador debe de imponer, existiendo ya en la actualidad varias medidas alternativas, de conformidad con los numerales 1 y 11 del artículo 77 de la Constitución, estableciéndose con ello, la constitucionalidad y legalidad del monitoreo por vigilancia electrónica, y un mecanismo para aplicar garantías penitenciarias, como los regímenes semi abierto y abierto, como garantías de prelibertad.

Pero debemos de indicar que se debe de incluir dentro del grupo de los vigilados electrónicamente, aquellas personas que son investigadas por delitos sexuales contra menores, los pedófilos y agresores sexuales, quienes son un peligro latente a nuestra infancia, catalogados por varios tratadistas como depredadores, el Estado debe también, sin que ello implique violación a sus derechos humanos, pero si un aseguramiento a los derechos de grupos de atención prioritario, por lo que el Estado estaría cumpliendo su máxima obligación que es de tutelar y proteger los derechos humanos. 


\section{La seguridad ciudadana frente al derecho a la defensa en libertad de los procesados}

Vol. 3, núm. 4., (2019)

Félix Arquímides Valdez Rivera

Pero, así como existen tesis favorables, existen tesis contrarias, entre los elementos que ciertos sectores catalogan como desfavorables tenemos:

- La medida en algunos países es considerada inconstitucional, ya que restringe el derecho de movilidad;

- Es una medida humillante, ya que estigmatiza a las personas que la utilizan;

- Las pulseras o brazaletes son réplicas de otras medidas alternativas como el arresto domiciliario;

- Existe dificultad para ser usadas, la poca familiaridad que existirá en el uso de una tecnología nueva, ya que puede resultar ser oneroso su implementación;

- Alto margen de fallos, perturbaciones, manipulaciones de los dispositivos de vigilancia electrónica;

- Abuso del derecho de control del Estado;

- Costos elevados para su implementación.

Pero muchas de estas antítesis, realmente no tiene fundamento técnico ni jurídico, son solo especulaciones de las que nunca faltan, pero la realidad es que la vigilancia electrónica es un medio óptimo para que las personas que infrinjan la ley penal puedan, acogerse a esta medida alternativa, que con un compromiso por parte de todos los actores puede asegurar acabar con el hacinamiento de las cárceles; siempre y cuando se cumplan los derechos fundamentales de estas personas.

Siendo esto como hemos establecido, una garantía de optimización en donde Ecuador ofrezca mejores procedimientos en todo el campo judicial, aplicando y acogiendo las 


\section{La seguridad ciudadana frente al derecho a la defensa en libertad de los procesados}

Vol. 3, núm. 4., (2019)

Félix Arquímides Valdez Rivera

recomendaciones de agencias internacionales u organismos supranacionales de derechos humanos, para aplicar mecanismos alternativos a la prisión preventiva; en esto radica el Estado garantista, el cual como expone el Dr. Augusto Durán Ponce: "Tal como exponía el Dr. Ferrajolí, el garantismo tiene tres acepciones: 1) modelo normativo de derecho; 2) teoría jurídica de la validez y de la efectividad; y, 3) filosofía política" (Durán Ponce, 2012), esto para satisfacer sin discriminación los derechos humanos y los postulados constante en el buen vivir.

\section{5.- Conclusiones y Recomendaciones}

El Texto Constitucional, consagra el Principio de Mínima Intervención Penal, en delitos que no representen mayor relevancia social, en donde seria, imperativa la medida cautelar de la prisión preventiva, permitiendo con ello la imposición de esta vigilancia electrónica a delitos de menor bagaje o denominado por el vulgo jurídico como delitos de bagatela, en donde debe de aplicarse medidas alternativas.

La problemática de la rehabilitación social ecuatoriana, no se soluciona construyendo nuevos Centros Carcelarios, sino utilizando métodos más idóneos y eficaces para la reinserción de la persona privada de su libertad, esto es, desde el inicio del proceso penal, el juzgador sepa valorar y utilizar la sana critica de todo lo que obra en el proceso, para que la prisión preventiva sea de ultima ratio, es decir, excepcional, y con esto evitar el hacinamiento en cárceles y penales.

Como se ha analizado en la presente, debe existir dentro del COIP, artículos más claros en cuanto a la implementación del dispositivo electrónico, para que posterior el Ministerio de 


\section{La seguridad ciudadana frente al derecho a la defensa en libertad de los procesados}

Vol. 3, núm. 4., (2019)

Félix Arquímides Valdez Rivera

Justicia, Derechos Humanos y Cultos, vía reglamento o directriz, canalice la correcta aplicación de dicho dispositivo.

Que el Estado ecuatoriano, debe ya de asumir con total entereza, la rehabilitación social del país, para ello ampliando la cobertura del dispositivo de vigilancia electrónica, y adquiriendo más unidades de monitoreo telemático, que estos incluyan vigilancia a personas que hayan sido sentenciadas por delitos sexuales, como en el caso de los pedófilos, quienes por su naturaleza, deben de estar vigilados, haciendo un símil a la legislación comparada, donde este tipo de injustos penales, son vigilados e inclusive se les prohíbe estar cerca de parques, escuelas, colegios, bibliotecas, y demás sitios donde son frecuentados por niños niñas y adolescentes.

Para ello se recomienda lo siguiente:

Proyecto de Ley Reformatoria al Código Orgánico Integral Penal, en base del sustento técnico y jurídico, ofrecido en la presente, en donde contengan normas que faciliten la aplicación de dispositivos de vigilancia electrónica, que sustituyan la medida de ultima ratio que es la prisión preventiva, asegurando la presencia de o los procesados a juicio, permitiendo que los procesados y sentenciados se acojan a esta institución jurídica de beneficio, como es el sistema de vigilancia electrónica.

Para ello, se debe sugerir la reforma al artículo 559, agregándole un inciso a continuación del actual inciso final: "La utilización del dispositivo electrónico, será aplicable en caso de delitos menores de cinco años, de igual forma para asegurar la no reincidencia del procesado, se implementara el dispositivo electrónico a las personas procesadas a quienes no se les ha aplicado 


\section{La seguridad ciudadana frente al derecho a la defensa en libertad de los procesados}

Vol. 3, núm. 4., (2019)

Félix Arquímides Valdez Rivera

medidas cautelares de ultima ratio, como la prisión preventiva, única y exclusivamente en delitos contra la libertad sexual y violencia intrafamiliar".

Para asegurar la correcta aplicación de la norma en la cual se solicita la reforma, el Consejo de la Judicatura, debe de implementar mesas de sensibilización para que los servidores judiciales se empoderen de la aplicación de las medidas cautelares, como la prisión preventiva que es excepcional, el artículo 536 y 537 del COIP, faculta al juzgador sustituir la medida cautelar establecida, en donde se establece el dispositivo de vigilancia electrónica, el arresto domiciliario con el dispositivo de vigilancia electrónica, mujeres embarazadas, enfermos incurables, discapacitados severas o de complejidad alta de enfermedades huérfanas, raras y catastróficas.

De igual forma que la Defensoría del Pueblo del Ecuador, cumpla su rol de tutelar, proteger y vigilar el debido proceso, para ello que se refuerce a las servidoras y servidores de la defensoría pública, para que den un óptimo servicio a la comunidad, para ello, la capacitación continua y que las resoluciones emitidas por esta Institución Nacional de Derechos Humanos, sean vinculantes en cuanto a la declaratoria de vulneración al debido proceso, para ello que el Consejo de la Judicatura inicie conversaciones con la Defensoría del Pueblo del Ecuador, para cumplir el mandato constitucional de que las instituciones deben coordinar acciones para tutelar y proteger los derechos humanos. 


\section{La seguridad ciudadana frente al derecho a la defensa en libertad de los \\ procesados}

Vol. 3, núm. 4., (2019)

Félix Arquímides Valdez Rivera

Cuadro No. 1.- Condenados que fueron beneficiados con la vigilancia electrónica en Estados Unidos de Norteamérica

\section{Condenados con dispositivos electrónicos}

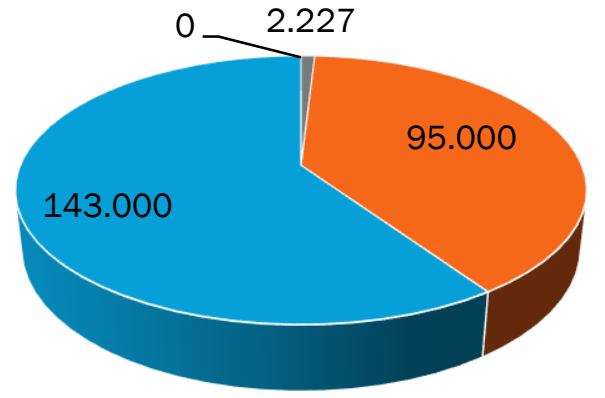

- 1988 - 1998 - 2009 -

Cuadro No. 2.- Condado de Cox (Estados Unidos), personas beneficiadas en el año 1989, tomado como muestra focalizada

\section{Monitoreo Telematico en el Condado de Cook, Illinois}

300.000

250.000

200.000

150.000

100.000

50.000

0

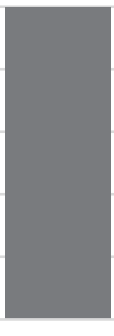

1989

- Columna1

\section{Bibliografía.}

Ardley, J. (2005). The Theory, Development and Application of Electronic Monitoring in Britain. Internet Journal of Criminology. Internacional. 


\section{La seguridad ciudadana frente al derecho a la defensa en libertad de los procesados}

Vol. 3, núm. 4., (2019)

Félix Arquímides Valdez Rivera

Caiado, N. (2013). Las grandes cuestiones éticas alrededor de la vigilancia eléctronica. Centro de Estudios en Seguridad Ciudadana de la Universidad de Chile.

Chávez Vallejo, G. (2013). Constitución para Servidores Públicos. Colección Nuevo Estado. Gobernabilidad, Derecho y Democracia. Quito: IAEN.

Diario El Universo. (21 de marzo de 2016). www.eluniverso.com. Obtenido de Brazalete electronico se utilizara en cuatro casos de fases de prelibertad: http://www.eluniverso.com/noticias/2016/03/21/nota/5480508/brazalete-electronico-seutilizara-cuatro-casos

Durán Ponce, A. (12 de agosto de 2012). www.derechoecuador.com. Obtenido de La Teoría Garantista:

http://www.derechoecuador.com/articulos/detalle/archive/doctrinas/derechoconstitucional /2012/08/16/la-teoria-garantista

Ecuador inmediato. (26 de 01 de 2016). www.ecuadorinmediato.com. Obtenido de Desde este mes probarán brazaletes electrónicos para PPL (Personas Privadas de la Libertad): www.ecuadorinmediato.com/brazaletes

ecuadorinmediato. (23 de septiembre de 2016). www.ecuadorinmediato.com. Obtenido de Desde este mes se probaran brazaletes electrónicos para PPL: http://www.ecuadorinmediato.com/index.php?module=Noticias\&func=news_user_view $\& \mathrm{id}=2818794144$

Ferrajolí, L. (1995).

Foucault, M. (2000). Vigilas y Castigar: el Nacimiento de la prisión. Madrid: Siglo XXI Editores.

Función del Sistema de Vigilancia Eelectronica en Colombia, C-185/11 (Corte Constitucional de Colombia 2011).

Galvis Trujillo, L. (2008). Comprensión de los Derechos Humanos. Bogotá: AuroraCorporación de Estudios y Públicaciones- CEP.

Garland, D., Wacquant, L., \& De Giorgi, A. (2006). El gobierno de la excedencia. Postfordismo y control de la multitud. Traficantes de sueños. Madrid : S.L/ VIRUS Editorial.

Goncalvez Machado, V. (2009). El Fracaso de la pena de prisión: alternativas y soluciones.

Poza Cisnero, M. (2001). Llas nuevas tecnologías en el ámbito penal.

Rodríguez Magariños, F. (2004-2005). Cárcel electrónica: Bases para la creación del sistema penitenciario del siglo XXI. 


\section{La seguridad ciudadana frente al derecho a la defensa en libertad de los}

procesados

Vol. 3, núm. 4., (2019)

Félix Arquímides Valdez Rivera

Romero Araque, T. (2016). Ánalisis comparativo de la aplicaicón del dispositivo de Vigilancia Electrónica en el Ecuador, en el caso de los procesados y condenados. Ambato, Ecuador.

Téllez Aguilar, A. (2011). Nuevas Penas y Medidas Alternativas a la Prisión.

$$
\text { (9) }(\mathcal{Q} \otimes(9
$$

RECONOCIMIENTO-NOCOMERCIAL-COMPARTIRIGUAL

CC BY-NC-SA

ESTA LICENCIA PERMITE A OTROS ENTREMEZCLAR, AJUSTAR Y CONSTRUIR A PARTIR DE SU OBRA CON FINES NO COMERCIALES, SIEMPRE Y CUANDO LE RECONOZCAN LA AUTORÍA Y SUS NUEVAS CREACIONES ESTÉN BAJO UNA LICENCIA CON LOS MISMOS TÉRMINOS. 